Article

\title{
Inverse Scattering and Soliton Solutions of Nonlocal Complex Reverse-Spacetime Modified Korteweg-de Vries Hierarchies
}

\author{
Liming Ling ${ }^{1}$ and Wen-Xiu Ma ${ }^{2,3,4,5, *(D)}$ \\ 1 School of Mathematics, South China University of Technology, Guangzhou 510640, China; linglm@scut.edu.cn \\ 2 Department of Mathematics, Zhejiang Normal University, Jinhua 321004, China \\ 3 Department of Mathematics, King Abdulaziz University, Jeddah 21589, Saudi Arabia \\ 4 Department of Mathematics and Statistics, University of South Florida, Tampa, FL 33620-5700, USA \\ 5 School of Mathematical and Statistical Sciences, Mafikeng Campus, North-West University, Private Bag X2046, \\ Mmabatho 2735, South Africa \\ * Correspondence: mawx@cas.usf.edu
}

Citation: Ling, L.; Ma, W.X. Inverse Scattering and Soliton Solutions of Nonlocal Complex Reverse-Spacetime Modified Korteweg-de Vries

Hierarchies. Symmetry 2021, 13, 512. https://doi.org/10.3390/sym13030512

Academic Editor: Attilio Maccari

Received: 4 March 2021

Accepted: 15 March 2021

Published: 21 March 2021

Publisher's Note: MDPI stays neutral with regard to jurisdictional claims in published maps and institutional affiliations.

Copyright: (c) 2021 by the authors. Licensee MDPI, Basel, Switzerland. This article is an open access article distributed under the terms and conditions of the Creative Commons Attribution (CC BY) license (https:// creativecommons.org/licenses/by/ $4.0 /)$.

\begin{abstract}
This paper aims to explore nonlocal complex reverse-spacetime modified Korteweg-de Vries $(\mathrm{mKdV})$ hierarchies via nonlocal symmetry reductions of matrix spectral problems and to construct their soliton solutions by the inverse scattering transforms. The corresponding inverse scattering problems are formulated by building the associated Riemann-Hilbert problems. A formulation of solutions to specific Riemann-Hilbert problems, with the jump matrix being the identity matrix, is established, where eigenvalues could equal adjoint eigenvalues, and thus $N$-soliton solutions to the nonlocal complex reverse-spacetime $\mathrm{mKdV}$ hierarchies are obtained from the reflectionless transforms.
\end{abstract}

Keywords: matrix spectral problem; soliton hierarchy; nonlocal PT symmetry; Riemann-Hilbert problem; Inverse scattering; soliton solution

MSC: 37K15; 35Q55; 37K40

\section{Introduction}

Nonlocal integrable equations have been shown to be significantly important in modern physics, and have already become one of the hottest topics in the field of integrable equations. Nonlocal partial differential equations occur in many contexts, including stochastic control, network science, fluid mechanics and quantum physics, indeed. Two types of nonlocal reverse-spacetime modified Korteweg-de Vries (mKdV) equations are presented upon taking nonlocal reductions [1]. One is recognized as "complex type" and the other is recognized as "real type", according to whether the conjugate of a potential is involved. The inverse scattering transforms to nonlocal integrable equations have been recently developed for the scalar case [2-7] and the multicomponent case [8], and soliton solutions have been generated from the Riemann-Hilbert problems with the identity jump matrix [8,9], via Darboux transformations [10-14] and the Hirota bilinear method [15,16]. Some other multicomponent generalizations [1,17] and nonlocal integrable equations $[5,18]$ were also presented. Based on the Ablowitz-Kaup-Newell-Segur (AKNS) spectral problem with multiple potentials, we would like to construct a class of $\mathrm{mKdV}$ hierarchies of nonlocal complex reverse-spacetime integrable equations and to discuss their inverse scattering transforms and soliton solutions from a perspective of Riemann-Hilbert problems.

The Riemann-Hilbert problems associated with matrix spectral problems are effectively used to solve integrable equations and particularly compute soliton solutions [19]. Various integrable equations, such as the multiple wave interaction equations [19], the general coupled nonlinear Schrödinger equations [20], the Harry Dym equation [21], the generalized Sasa-Satsuma equation [22], the multicomponent modified Korteweg-de Vries 
(mKdV) equations [23] and soliton hierarchies [24], have been studied via building and solving their associated Riemann-Hilbert problems.

Let us review the general procedure for building Riemann-Hilbert problems on the real line $[23,24]$ as follows. We begin with a pair of matrix spectral problems:

$$
-i \phi_{x}=U \phi,-i \phi_{t}=V \phi, U=A(\lambda)+P(u, \lambda), V=B(\lambda)+Q(u, \lambda),
$$

where $u$ is a potential, $\lambda$ is a spectral parameter, $\phi$ is a square matrix eigenfunction, $i$ denotes the unit imaginary number, and we often assume that $A$ and $B$ are constant and commuting diagonal square matrices, and $P$ and $Q$ are square matrices being trace-less. The compatibility condition of the above two matrix spectral problems, that is, the zero curvature equation,

$$
U_{t}-V_{x}+i[U, V]=0,
$$

where $[U, V]=U V-V U$, presents an integrable equation. To formulate an associated Riemann-Hilbert problem, we adopt an equivalent pair of matrix spectral problems:

$$
\psi_{x}=i[A(\lambda), \psi]+\check{P}(u, \lambda) \psi, \psi_{t}=i[B(\lambda), \psi]+\check{Q}(u, \lambda) \psi,
$$

where $\check{P}=i P, \check{Q}=i Q$, and $\psi$ is assumed to be a square matrix eigenfunction. Clearly, the equivalence is guaranteed by the commutativity of the two constant matrices $A$ and $B$. The relation of $\psi$ to $\phi$ is given by

$$
\psi=\phi E_{g}^{-1}, E_{g}=\mathrm{e}^{i A(\lambda) x+i B(\lambda) t} .
$$

To obtain the uniqueness of matrix eigenfunctions, we normally take the asymptotic conditions

$$
\psi^{ \pm} \rightarrow I, \text { when } x, t \rightarrow \pm \infty,
$$

where $I$ denotes the identity matrix. From those two eigenfunction matrices $\psi^{ \pm}$, we pick the entries to construct two generalized matrix Jost functions $T^{ \pm}(x, t, \lambda)$, which are analytical in the upper and lower half-planes $\mathbb{C}^{ \pm}$, and continuous in the closed upper and lower halfplanes $\overline{\mathbb{C}}^{ \pm}$, respectively. Then, by using $T^{+}$and $T^{-}$, we can formulate a Riemann-Hilbert problem on the real axis associated with the integrable Equation (2):

$$
G^{+}(x, t, \lambda)=G^{-}(x, t, \lambda) G_{0}(x, t, \lambda), \lambda \in \mathbb{R},
$$

where $G_{0}$ is called the jump matrix, and $G^{ \pm}$are two generalized matrix Jost functions being unimodular. From such an associated Riemann-Hilbert problem, we can generate an inverse scattering transform.

Observe that $\psi^{-}$and $\psi^{+}$, the two matrix eigenfunctions, are linearly dependent, and thus, we can have the relation

$$
\psi^{-} E_{g}=\psi^{+} E_{g} S(\lambda),
$$

where $S(\lambda)$ is usually called the scattering matrix of the given matrix spectral problems (1). The jump matrix $G_{0}$ carries all basic scattering data from the corresponding scattering matrix $S(\lambda)$. The Riemann-Hilbert problem (6) can be solved through the Sokhotski-Plemelj formula [25]:

$$
G(\lambda)=I+\frac{1}{2 \pi i} \int_{-\infty}^{\infty} \frac{\left(G^{+}-G^{-}\right)(\xi)}{\xi-\lambda} d \xi,
$$

if $G$ has no pole, and the resulting solutions can be used to build the needed generalized matrix Jost functions for retrieving the potential in the original matrix spectral problems, which solves the given integrable equation. The whole procedure forms an inverse scattering transform. Soliton solutions are constructed from the specific Riemann-Hilbert problem with $G_{0}=I$, that is, the identity jump matrix, or alternatively, from the reflectionless inverse scattering transform. 
The rest of the paper is organized as follows-in Section 2, we carry out a kind of nonlocal reverse-spacetime reductions and construct nonlocal integrable hierarchies of complex reverse-spacetime $\mathrm{mKdV}$ equations, on the basis of the multicomponent AKNS soliton hierarchy. In Section 3, we discuss their corresponding inverse scattering transforms with the help of the associated Riemann-Hilbert problems. In Section 4, we first present a solution formulation for specific Riemann-Hilbert problems over the real line, whose jump matrix is the identity. Then, through such a formulation of solutions, we compute soliton solutions for the nonlocal complex reverse-spacetime mKdV hierarchies. In our analysis, eigenvalues could equal adjoint eigenvalues, which has never happened in the local theory. In the final section, we present a conclusion, along with a few futuristic remarks.

\section{Nonlocal Symmetric Reductions and Nonlocal mKdV Hierarchies}

\subsection{Multicomponent AKNS Hierarchy}

Let us recall the multicomponent AKNS hierarchy here for convenience of reference. Let $n \geq 1$ be an arbitrary integer, $\alpha_{1}$ and $\alpha_{2}$, arbitrary but different constants, and $I_{n}$, the identity matrix of size $n$. The multicomponent AKNS matrix spectral problem is given by (see, e.g., [26]):

$$
-i \phi_{x}=U \phi=U(u, \lambda) \phi, U=\left(U_{j l}\right)_{(n+1) \times(n+1)}=\left[\begin{array}{cc}
\alpha_{1} \lambda & p \\
q & \alpha_{2} \lambda I_{n}
\end{array}\right],
$$

in which $\lambda$ denotes a spectral parameter and $u$ stands for a $2 n$-dimensional potential:

$$
u=\left(p, q^{T}\right)^{T}, p=\left(p_{1}, \cdots, p_{n}\right), q=\left(q_{1}, \cdots, q_{n}\right)^{T} .
$$

We remark that when $p_{j}=q_{j}=0,2 \leq j \leq n,(9)$ is reduced to the standard AKNS spectral problem [27].

Let us assume [26] that a solution $W$ to $W_{x}=i[U, W]$ takes the form

$$
W=\left[\begin{array}{ll}
a & b \\
c & d
\end{array}\right]=\sum_{m=0}^{\infty} W_{m} \lambda^{-m}, W_{m}=W_{m}(u)=\left[\begin{array}{ll}
a^{[m]} & b^{[m]} \\
c^{[m]} & d^{[m]}
\end{array}\right], m \geq 0,
$$

in which $b^{[m]}, c^{[m]}$ and $d^{[m]}$, denoted as

$$
b^{[m]}=\left(b_{1}^{[m]}, b_{2}^{[m]}, \cdots, b_{n}^{[m]}\right), c^{[m]}=\left(c_{1}^{[m]}, c_{2}^{[m]}, \cdots, c_{n}^{[m]}\right)^{T}, d^{[m]}=\left(d_{j l}^{[m]}\right)_{n \times n}, m \geq 0,
$$

are determined recursively by

$$
\begin{gathered}
b^{[0]}=0, c^{[0]}=0, a_{x}^{[0]}=0, d_{x}^{[0]}=0, \\
b^{[m+1]}=\frac{1}{\alpha}\left(-p d^{[m]}+a^{[m]} p-i b_{x}^{[m]}\right), m \geq 0, \\
c^{[m+1]}=\frac{1}{\alpha}\left(-d^{[m]} q+q a^{[m]}+i c_{x}^{[m]}\right), m \geq 0, \\
a_{x}^{[m]}=-i\left(b^{[m]} q-p c^{[m]}\right), d_{x}^{[m]}=i\left(q b^{[m]}-c^{[m]} p\right), m \geq 1,
\end{gathered}
$$

where $\alpha=\alpha_{1}-\alpha_{2}$. Together with the initial data:

$$
a^{[0]}=\beta_{1}, d^{[0]}=\beta_{2} I_{n}
$$

$\beta_{1}, \beta_{2}$ being different but arbitrary constants, and by taking zero constants of integration in (16), that is,

$$
\left.W_{m}\right|_{u=0}=0, m \geq 1,
$$


the recursive relations in (13)-(16) uniquely determine all matrices $W_{m}, m \geq 1$. Particularly, we obtain

$$
\begin{gathered}
b_{j}^{[1]}=\frac{\beta}{\alpha} p_{j,} c_{j}^{[1]}=\frac{\beta}{\alpha} q_{j}, a^{[1]}=0, d_{j l}^{[1]}=0 ; \\
b_{j}^{[2]}=-\frac{\beta}{\alpha^{2}} i p_{j, x}, c_{j}^{[2]}=\frac{\beta}{\alpha^{2}} i q_{j, x}, a^{[2]}=-\frac{\beta}{\alpha^{2}} p q, d_{j l}^{[2]}=\frac{\beta}{\alpha^{2}} p_{l} q_{j} ; \\
\left\{\begin{array}{l}
b_{j}^{[3]}=-\frac{\beta}{\alpha^{3}}\left(p_{j, x x}+2 p q p_{j}\right), c_{j}^{[3]}=-\frac{\beta}{\alpha^{3}}\left(q_{j, x x}+2 p q q_{j}\right), \\
a^{[3]}=-\frac{\beta}{\alpha^{3}} i\left(p q_{x}-p_{x} q\right), d_{j l}^{[3]}=-\frac{\beta}{\alpha^{3}} i\left(p_{l, x} q_{j}-p_{l} q_{j, x}\right) ; \\
b_{j}^{[4]}=\frac{\beta}{\alpha^{4}} i\left(p_{j, x x x}+3 p q p_{j, x}+3 p_{x} q p_{j}\right), \\
c_{j}^{[4]}=-\frac{\beta}{\alpha^{4}} i\left(q_{j, x x x}+3 p q_{x} q_{j}+3 p q q_{j, x}\right), \\
a^{[4]}=\frac{\beta}{\alpha^{4}}\left[3(p q)^{2}+p q_{x x}+p_{x x} q-p_{x} q_{x}\right], \\
d_{j l}^{[4]}=-\frac{\beta}{\alpha^{4}}\left(3 p_{l} p q q_{j}+p_{l, x x} q_{j}+p_{l} q_{j, x x}-p_{l, x} q_{j, x}\right) ;
\end{array}\right.
\end{gathered}
$$

where $\beta=\beta_{1}-\beta_{2}$ and $1 \leq j, l \leq n$. A recurrence relation for determining $b^{[m]}$ and $c^{[m]}$ is given by

$$
\left[\begin{array}{c}
c^{[m+1]} \\
b^{[m+1] T}
\end{array}\right]=\Psi\left[\begin{array}{c}
c^{[m]} \\
b^{[m] T}
\end{array}\right], m \geq 1,
$$

in which $\Psi$ is the following $2 n \times 2 n$ matrix operator

$$
\Psi=\frac{i}{\alpha}\left[\begin{array}{cc}
\left(\partial+\sum_{j=1}^{n} q_{j} \partial^{-1} p_{j}\right) I_{n}+q \partial^{-1} p & -\left(q \partial^{-1} q^{T}\right)^{T}-q \partial^{-1} q^{T} \\
\left(p^{T} \partial^{-1} p\right)^{T}+p^{T} \partial^{-1} p & -\left(\partial+\sum_{j=1}^{n} p_{j} \partial^{-1} q_{j}\right) I_{n}-p^{T} \partial^{-1} q^{T}
\end{array}\right] .
$$

Upon introducing the temporal matrix spectral problems:

$$
\left\{\begin{array}{l}
-i \phi_{t}=V^{[r]} \phi=V^{[r]}(u, \lambda) \phi, \\
V^{[r]}=\left(V_{j l}^{[r]}\right)_{(n+1) \times(n+1)}=\sum_{l=0}^{r} W_{l} \lambda^{r-l}, r \geq 0,
\end{array}\right.
$$

we see that the compatibility conditions in the system of (9) and (21), namely, the following zero curvature equations

$$
U_{t}-V_{x}^{[r]}+i\left[U, V^{[r]}\right]=0, r \geq 0
$$

lead to the so-called multicomponent AKNS soliton hierarchy:

$$
u_{t}=\left[\begin{array}{l}
p^{T} \\
q
\end{array}\right]_{t}=K_{r}=i\left[\begin{array}{c}
\alpha b^{[r+1] T} \\
-\alpha c^{[r+1]}
\end{array}\right]=i J G_{r}, r \geq 0
$$

where

$$
J=\left[\begin{array}{cc}
0 & \alpha I_{n} \\
-\alpha I_{n} & 0
\end{array}\right], G_{r}=\left[\begin{array}{c}
c^{[r+1]} \\
b^{[r+1] T}
\end{array}\right], r \geq 0
$$

One of the nonlinear members $(r=3)$ in the hierarchy (23) is the standard mKdV equations:

$$
p_{t}=-\frac{\beta}{\alpha^{3}}\left(p_{x x x}+3 p q p_{x}+3 p_{x} q p\right), q_{t}=-\frac{\beta}{\alpha^{3}}\left(q_{x x x}+3 q_{x} p q+3 q p q_{x}\right) \text {. }
$$


The multicomponent AKNS soliton hierarchy (23) possesses a Hamiltonian formulation, which can be obtained from an application of the trace identity [28], or more generally, of the variational identity [29]:

$$
u_{t}=K_{r}=J \frac{\delta \tilde{H}_{r+1}}{\delta u}, r \geq 1
$$

where

$$
\tilde{H}_{s}=-\frac{i}{s} \int\left(\alpha_{1} a^{[s+1]}+\alpha_{2} \sum_{j=1}^{n} d_{j j}^{[s+1]}\right) d x, s \geq 1 .
$$

The integro-differential operator $\Phi=\Psi^{\dagger}$ presents a hereditary recursion operator for the whole soliton hierarchy (23). For each fixed $r \geq 1$, adjoint symmetry constraints (or equivalently, symmetry constraints induced by the Hamiltonian structure) can break the $r$ th multicomponent AKNS equations into two commuting finite-dimensional Hamiltonian systems being Liouville integrable, which present involutive solutions [26,30].

\subsection{Nonlocal Reverse-Spacetime $m K d V$ Hierarchies}

We denote the potential matrix $P$ by

$$
P=\left[\begin{array}{ll}
0 & p \\
q & 0
\end{array}\right]
$$

For convenience, we write $A(x, t, \lambda)=A(u(x, t), \lambda), A^{\dagger}(f(x, t, \lambda))=(A(f(x, t, \lambda)))^{\dagger}$, and $A^{-1}(f(x, t, \lambda))=(A(f(x, t, \lambda)))^{-1}$, for a given matrix $A$ depending on the potential $u$ and a function $f$.

The classical local reductions [31] motivates us to make a kind of special nonlocal reverse-spacetime symmetric reductions for the spectral matrix:

$$
U^{\dagger}\left(-x,-t,-\lambda^{*}\right)=-C U(x, t, \lambda) C^{-1} \text { with } C=\left[\begin{array}{cc}
1 & 0 \\
0 & \Sigma
\end{array}\right], \Sigma^{\dagger}=\Sigma,
$$

which equivalently yields the following reduction on $P$ :

$$
P^{\dagger}(-x,-t)=-C P(x, t) C^{-1}
$$

Here and in what follows, $*$ is the complex conjugate, + stands for the Hermitian transpose, and $\Sigma$ denotes a constant invertible Hermitian matrix.

It is easy to see that (30) equivalently tells

$$
q(x, t)=-\Sigma^{-1} p^{\dagger}(-x,-t) .
$$

Under such a kind of nonlocal reductions, we can take the vector function $c$ in $W$ as

$$
c(x, t, \lambda)=-\Sigma^{-1} b^{\dagger}\left(-x,-t,-\lambda^{*}\right),
$$

and those two reduction relations guarantee that

$$
\left\{\begin{array}{l}
a^{*}\left(-x,-t,-\lambda^{*}\right)=-a(x, t, \lambda), \\
d^{\dagger}\left(-x,-t,-\lambda^{*}\right) \Sigma=-\Sigma d(x, t, \lambda),
\end{array}\right.
$$

where $a, b, c$ and $d$ are the entries of $W$. To be precise, we obtain

$$
\left\{\begin{array}{l}
\left(a^{[m]}\right)^{*}(-x,-t)=(-1)^{m+1} a^{[m]}(x, t), \\
\left(b^{[m]}\right)^{\dagger}(-x,-t)=(-1)^{m+1} \Sigma c^{[m]}(x, t), \quad m \geq 1, \\
\left(d^{[m]}\right)^{\dagger}(-x,-t) \Sigma=(-1)^{m+1} \Sigma d^{[m]}(x, t),
\end{array}\right.
$$


where $a^{[m]}, b^{[m]}, c^{[m]}$ and $d^{[m]}, m \geq 1$, are defined as in (11). It then follows that

$$
\left(V^{[2 m+1]}\right)^{\dagger}\left(-x,-t,-\lambda^{*}\right)=-C V^{[2 m+1]}(x, t, \lambda) C^{-1}, m \geq 0,
$$

where $V^{[2 m+1]}$ is defined as in (21).

Now, based on (29) and (35), we can determine that all the nonlocal reductions in (29) generate no additional condition on the compatibility conditions in the previous spatial and temporal matrix spectral problems (9) and (21), when $r=2 m+1$. To conclude, under the nonlocal reverse-spacetime reductions defined by (29), the half hierarchies of the equations in (23) with $r=2 m+1$ are reduced to the nonlocal complex reverse-spacetime PT-symmetric mKdV hierarchies:

$$
p_{t}=X_{m}=\left.K_{2 m+1,1}\right|_{q=-\Sigma^{-1} p^{\dagger}(-x,-t)}, m \geq 0,
$$

where $K_{r}=\left(K_{r, 1}^{T}, K_{r, 2}^{T}\right)^{T}=i\left(\alpha b^{(r+1)},-\alpha c^{(r+1) T}\right)^{T}, r \geq 0$. We remark that those soliton hierarchies with all integers $n \geq 1$ are generated from the following matrix spectral problems

$$
-i \phi_{x}=U \phi=U(u, \lambda) \phi,-i \phi_{t}=V^{[2 m+1]} \phi=V^{[2 m+1]}(u, \lambda) \phi, m \geq 0,
$$

in which the involved Lax pairs read

$$
U=\lambda \Lambda+P, V^{[2 m+1]}=\lambda^{2 m+1} \Omega+Q_{2 m+1}, m \geq 0,
$$

with $\Lambda=\operatorname{diag}\left(\alpha_{1}, \alpha_{2} I_{n}\right), \Omega=\operatorname{diag}\left(\beta_{1}, \beta_{2} I_{n}\right), P$ being defined by (28), and

$$
Q_{2 m+1}=\sum_{l=1}^{2 m+1} \lambda^{2 m-l+1}\left[\begin{array}{ll}
a^{[l]} & b^{[l]} \\
c^{[l]} & d^{[l]}
\end{array}\right], m \geq 0 .
$$

Moreover, each hierarchy has infinitely many commuting Lie symmetries $\left\{X_{k}\right\}_{k=0}^{\infty}$ and infinitely many commuting local conserved functionals $\left\{\left.\tilde{H}_{2 k+2}\right|_{q=-\Sigma^{-1}} p^{\dagger}(-x,-t)\right\}_{k=0}^{\infty}$.

The case of $m=1$ gives the following nonlocal complex reverse-spacetime multicomponent $\mathrm{mKdV}$ equations:

$$
\begin{aligned}
p_{t}(x, t)= & -\frac{\beta}{\alpha^{3}}\left[p_{x x x}(x, t)-3 p(x, t) \Sigma^{-1} p^{\dagger}(-x,-t) p_{x}(x, t)\right. \\
& \left.-3 p_{x}(x, t) \Sigma^{-1} p^{\dagger}(-x,-t) p(x, t)\right],
\end{aligned}
$$

in which $\Sigma$ denotes an arbitrary invertible Hermitian matrix. Further, the subcase of $n=1$ can yield two scalar equation examples presented in [2]:

$$
p_{t}(x, t)=p_{x x x}(x, t)-6 \sigma p(x, t) p^{*}(-x,-t) p_{x}(x, t), \sigma= \pm 1,
$$

and the case of $n=2$ can engender a new example of coupled equations:

$$
\left\{\begin{array}{l}
p_{1, t}(x, t)=p_{1, x x x}(x, t)+c_{1}\left(p_{1}^{2}\right)_{x}(x, t) p_{1}^{*}(-x,-t)+c_{2}\left(p_{1} p_{2}\right)_{x}(x, t) p_{2}^{*}(-x,-t), \\
p_{2, t}(x, t)=p_{2, x x x}(x, t)+c_{1}\left(p_{1} p_{2}\right)_{x}(x, t) p_{1}^{*}(-x,-t)+c_{2}\left(p_{2}^{2}\right)_{x}(x, t) p_{2}^{*}(-x,-t),
\end{array}\right.
$$

where $c_{1}$ and $c_{2}$ are arbitrary nonzero real numbers. Such nonlocal mKdV equations possess the PT symmetry that if $p(x, t)$ is a solution, so is $p^{\dagger}(-x,-t)$. They could serve as new mathematical models in many physical situations, where the nonlinearity must be taken into account to cubic order, like the local $\mathrm{mKdV}$ equations, which can describe, for example, nonlinear propagations of electro-hydrodynamic surface wave and heat pulses in solids. 


\section{Inverse Scattering Transforms}

In what follows, we analyze the scattering and inverse scattering transforms for the nonlocal complex reverse-spacetime PT-symmetric mKdV hierarchies in (36) through using the Riemann-Hilbert technique [19] (also refer [32,33]). We discuss the inverse scattering transforms through building and then solving the associated Riemann-Hilbert problems. In this section, we always assume that $q$ is determined by (31).

\subsection{Property of Eigenfunctions}

Let us suppose that, for the matrix spectral problems in (37), all the involved potential functions sufficiently rapidly vanish at infinity, that is, as $x \rightarrow \pm \infty$ or $t \rightarrow \pm \infty$, and thus, one can have the asymptotic behavior: $\phi \sim \mathrm{e}^{i \lambda \Lambda x+i \lambda^{2 m+1} \Omega t}$, as $x$ or $t \rightarrow \pm \infty$. The eigenfunction transformation

$$
\phi=\psi E_{g}, E_{g}=\mathrm{e}^{i \lambda \Lambda x+i \lambda^{2 m+1} \Omega t},
$$

engenders the canonical normalization for $\psi: \psi \rightarrow I_{n+1}$, as $x$ or $t \rightarrow \pm \infty$. Upon defining $\breve{P}=i P$ and $\breve{Q}_{2 m+1}=i Q_{2 m+1}$, the equivalent pair of spatial and temporal matrix spectral problems to (37) is given by

$$
\begin{gathered}
\psi_{x}=i \lambda[\Lambda, \psi]+\check{P} \psi, \\
\psi_{t}=i \lambda^{2 m+1}[\Omega, \psi]+\check{Q}_{2 m+1} \psi .
\end{gathered}
$$

Because of $\operatorname{tr}(\check{P})=\operatorname{tr}\left(\check{Q}_{2 m+1}\right)=0$, one can get

$$
\operatorname{det} \psi=1,
$$

by using a generalized Liouville's formula [34].

For the spatial matrix spectral problems in (37) and (43), the adjoint counterparts are as follows:

$$
i \tilde{\phi}_{x}=\tilde{\phi} U
$$

and

$$
i \tilde{\psi}_{x}=\lambda[\tilde{\psi}, \Lambda]+\tilde{\psi} P,
$$

respectively. Observe that the inverse matrices, $\tilde{\phi}=\phi^{-1}$ and $\tilde{\psi}=\psi^{-1}$, solves the above adjoint matrix spectral problems, if $\phi$ and $\psi$ solves the spatial matrix spectral problems in (37) and (43). All pairs of adjoint spectral problems, and equivalent adjoint spectral problems, create no new condition, beyond the nonlocal complex reverse-spacetime PTsymmetric $\mathrm{mKdV}$ hierarchies of equations in (36).

Let us consider a matrix eigenfunction $\psi(\lambda)$ of the spatial matrix spectral problem (43) associated with an eigenvalue $\lambda$. Apparently, $C \psi^{-1}(x, t, \lambda)$ solves the adjoint spatial spectral problem with the same eigenvalue $\lambda$. Moreover, under the nonlocal symmetric reductions in (30), one can compute that

$$
\begin{aligned}
& i\left[\psi^{\dagger}\left(-x,-t,-\lambda^{*}\right) C\right]_{x}=i\left[-\left(\psi_{x}\right)^{\dagger}\left(-x,-t,-\lambda^{*}\right) C\right] \\
& =-i\left\{(-i)(-\lambda)\left[\psi^{\dagger}\left(-x,-t,-\lambda^{*}\right), \Lambda\right]+(-i) \psi^{\dagger}\left(-x,-t,-\lambda^{*}\right) P^{\dagger}(-x,-t)\right\} C \\
& =\lambda\left[\psi^{\dagger}\left(-x,-t,-\lambda^{*}\right), \Lambda\right] C+\psi^{\dagger}\left(-x,-t,-\lambda^{*}\right) C\left[-C^{-1} P^{\dagger}(-x,-t) C\right] \\
& =\lambda\left[\psi^{\dagger}\left(-x,-t,-\lambda^{*}\right) C, \Lambda\right]+\psi^{\dagger}\left(-x,-t,-\lambda^{*}\right) C P(x, t),
\end{aligned}
$$

and thus, a new matrix function

$$
\tilde{\psi}(x, t, \lambda):=\psi^{\dagger}\left(-x,-t,-\lambda^{*}\right) C,
$$

gives a new matrix adjoint eigenfunction with the same given eigenvalue $\lambda$, namely, the matrix function $\psi^{\dagger}\left(-x,-t,-\lambda^{*}\right) C$ presents a solution to the adjoint spatial matrix spectral problem (47). 
Then, by checking the imposed asymptotic behaviors at infinity for $\psi$, the uniqueness of solutions tells

$$
\psi^{\dagger}\left(-x,-t,-\lambda^{*}\right)=C \psi^{-1}(x, t, \lambda) C^{-1},
$$

if the matrix eigenfunction $\psi \rightarrow I_{n+1}$, as $x$ or $t \rightarrow \infty$ or $-\infty$. Therefore, it follows that when $\lambda$ presents an eigenvalue of (43) (or (47)), so does $-\lambda^{*}$, and the eigenfunction property (49) is satisfied.

\subsection{Riemann-Hilbert Problems}

In this subsection, we construct a class of associated Riemann-Hilbert problems with the space variable $x$. It is pointed out that the generating procedure for Riemann-Hilbert problems is the same as the one for the local PT-symmetric mKdV equations [23,31]. We just present the associated Riemann-Hilbert problems briefly below for subsequent discussions.

In order to state Riemann-Hilbert problems precisely, let us assume that

$$
\alpha=\alpha_{1}-\alpha_{2}<0, \beta=\beta_{1}-\beta_{2}<0
$$

In this way, we can recognize what entries of matrix eigenfunctions to select. In the setup of the scattering problem, we define the two needed matrix eigenfunctions $\psi^{ \pm}(x, \lambda)$ of the spectral problems (43) by imposing the asymptotic conditions

$$
\psi^{ \pm} \rightarrow I_{n+1}, \text { as } x \rightarrow \pm \infty
$$

respectively. The property (45) implies that the determinants $\operatorname{det} \psi^{ \pm}=1$ for all values $x \in \mathbb{R}$. Because

$$
\phi^{ \pm}=\psi^{ \pm} E, E=\mathrm{e}^{i \lambda \Lambda x}
$$

are all matrix eigenfunctions of the spectral problems (37), the two matrices must be linearly dependent. As a consequence, ones have

$$
\psi^{-} E=\psi^{+} E S(\lambda), \lambda \in \mathbb{R}
$$

in which $S(\lambda)=\left(s_{j l}\right)_{(n+1) \times(n+1)}$ is usually called the scattering matrix of the associated spectra problem. Note that we have $\operatorname{det} S(\lambda)=1$, on account of the determinants $\operatorname{det} \psi^{ \pm}=1$.

One can turn the spatial spectral problem in (37) into the second kind of Volterra integral equations for $\psi^{ \pm}[19,35]$ :

$$
\begin{aligned}
& \psi^{-}(x, \lambda)=I_{n+1}+\int_{-\infty}^{x} \mathrm{e}^{i \lambda \Lambda(x-y)} \check{P}(y) \psi^{-}(\lambda, y) \mathrm{e}^{i \lambda \Lambda(y-x)} d y, \\
& \psi^{+}(x, \lambda)=I_{n+1}-\int_{x}^{\infty} \mathrm{e}^{i \lambda \Lambda(x-y)} \check{P}(y) \psi^{+}(\lambda, y) \mathrm{e}^{i \lambda \Lambda(y-x)} d y,
\end{aligned}
$$

where we have used the asymptotic conditions (51). Let us express

$$
\psi^{ \pm}=\left(\psi_{1}^{ \pm}, \psi_{2}^{ \pm}, \cdots, \psi_{n+1}^{ \pm}\right)
$$

that is, $\psi_{j}^{ \pm}$denote the $j$ th columns of $\phi^{ \pm}(1 \leq j \leq n+1)$, and

$$
\tilde{\psi}^{ \pm}=\left(\psi^{ \pm}\right)^{-1}=\left[\begin{array}{c}
\tilde{\psi}^{ \pm, 1} \\
\tilde{\psi}^{ \pm, 2} \\
\vdots \\
\tilde{\psi}^{ \pm, n+1}
\end{array}\right]
$$


namely, $\tilde{\psi}^{ \pm, j}$ denote the $j$ th rows of $\tilde{\psi}^{ \pm}(1 \leq j \leq n+1)$. In this way, the general theory of Volterra integral equations (or more generally, the analytic Fredholm theory) can tell that one can make the required generalized matrix Jost function $T^{+}$to be

$$
T^{+}=T^{+}(x, \lambda)=\left(\psi_{1}^{-}, \psi_{2}^{+}, \cdots, \psi_{n+1}^{+}\right)=\psi^{-} H_{1}+\psi^{+} H_{2},
$$

which is analytic in $\mathbb{C}^{+}$and continuous in $\overline{\mathbb{C}}^{+}$as a function of $\lambda$; and make the required generalized matrix Jost function $T^{-}$to be

$$
T^{-}=\left[\begin{array}{c}
\tilde{\psi}^{-, 1} \\
\tilde{\psi}^{+, 2} \\
\vdots \\
\tilde{\psi}^{+, n+1}
\end{array}\right]=H_{1} \tilde{\psi}^{-}+H_{2} \tilde{\psi}^{+}=H_{1}\left(\psi^{-}\right)^{-1}+H_{2}\left(\psi^{+}\right)^{-1},
$$

which is analytic in $\mathbb{C}^{-}$and continuous in $\overline{\mathbb{C}}^{-}$as a function of $\lambda$. Here we define $H_{1}$ and $\mathrm{H}_{2}$ to be

$$
H_{1}=\operatorname{diag}(1, \underbrace{0, \cdots, 0}_{n}), H_{2}=\operatorname{diag}(0, \underbrace{1, \cdots, 1}_{n}) .
$$

Now, let us formulate the associated Riemann-Hilbert problems for the nonlocal reverse-spacetime PT-symmetric $\mathrm{mKdV}$ hierarchies in (36), based on those generalized matrix Jost functions, $T^{+}$and $T^{-}$. Obviously, from both $\operatorname{det} \psi^{ \pm}=1$ and the scattering relation (53) between the two matrix eigenfunctions $\psi^{+}$and $\psi^{-}$, one obtains

$$
\operatorname{det} T^{+}(x, \lambda)=s_{11}(\lambda), \operatorname{det} T^{-}(x, \lambda)=\hat{s}_{11}(\lambda),
$$

in which we denote $S^{-1}(\lambda)=(S(\lambda))^{-1}=\left(\hat{s}_{j l}\right)_{(n+1) \times(n+1)}$. Then, it follows that

$$
\left\{\begin{array}{l}
\lim _{x \rightarrow \infty} T^{+}(x, \lambda)=\left[\begin{array}{cc}
s_{11}(\lambda) & 0 \\
0 & I_{n}
\end{array}\right], \lambda \in \overline{\mathbb{C}}^{+}, \\
\lim _{x \rightarrow \infty} T^{-}(x, \lambda)=\left[\begin{array}{cc}
\hat{s}_{11}(\lambda) & 0 \\
0 & I_{n}
\end{array}\right], \lambda \in \overline{\mathbb{C}}^{-},
\end{array}\right.
$$

and further we can define two unimodular generalized matrix Jost functions as follows:

$$
\left\{\begin{array}{l}
G^{+}(x, \lambda)=T^{+}(x, \lambda)\left[\begin{array}{cc}
s_{11}^{-1}(\lambda) & 0 \\
0 & I_{n}
\end{array}\right], \lambda \in \overline{\mathbb{C}}^{+}, \\
\left(G^{-}\right)^{-1}(x, \lambda)=\left[\begin{array}{cc}
\hat{s}_{11}^{-1}(\lambda) & 0 \\
0 & I_{n}
\end{array}\right] T^{-}(x, \lambda), \lambda \in \overline{\mathbb{C}}^{-} .
\end{array}\right.
$$

These matrices constitute the required matrix Riemann-Hilbert problems on the real axis for the nonlocal complex reverse-spacetime PT-symmetric mKdV hierarchies in (36):

$$
G^{+}(x, \lambda)=G^{-}(x, \lambda) G_{0}(x, \lambda), \lambda \in \mathbb{R},
$$

in which by (53), the jump matrix $G_{0}$ is determined by

$$
G_{0}(x, \lambda)=E\left[\begin{array}{cc}
\hat{s}_{11}^{-1}(\lambda) & 0 \\
0 & I_{n}
\end{array}\right] \tilde{S}(\lambda)\left[\begin{array}{cc}
s_{11}^{-1}(\lambda) & 0 \\
0 & I_{n}
\end{array}\right] E^{-1} .
$$

The matrix $\tilde{S}(\lambda)$ involved in $G_{0}$ possesses the following matrix factorization:

$$
\tilde{S}(\lambda)=\left(H_{1}+H_{2} S(\lambda)\right)\left(H_{1}+S^{-1}(\lambda) H_{2}\right),
$$


and it can be worked out:

$$
\tilde{S}(\lambda)=\left(\tilde{s}_{j l}\right)_{(n+1) \times(n+1)}=\left[\begin{array}{ccccc}
1 & \hat{s}_{12} & \hat{s}_{13} & \cdots & \hat{s}_{1, n+1} \\
s_{21} & 1 & 0 & \cdots & 0 \\
s_{31} & 0 & 1 & \ddots & \vdots \\
\vdots & \vdots & \ddots & \ddots & 0 \\
s_{n+1,1} & 0 & \cdots & 0 & 1
\end{array}\right] .
$$

Again, following the second kind of Volterra integral equations (54) and (55), one can derive the canonical normalization conditions:

$$
G^{ \pm}(x, \lambda) \rightarrow I_{n+1}, \text { as } \lambda \in \overline{\mathbb{C}}^{ \pm} \rightarrow \infty,
$$

for the resulting Riemann-Hilbert problems on the real line. Furthermore, by the property of eigenfunctions in (49), one can determine that

$$
\left(T^{+}\right)^{\dagger}\left(-x,-t,-\lambda^{*}\right)=C T^{-}(x, t, \lambda) C^{-1},
$$

and hence, we see that the above jump matrix $G_{0}$ satisfies the involution property:

$$
G_{0}^{\dagger}\left(-x,-t,-\lambda^{*}\right)=C G_{0}(x, t, \lambda) C^{-1},
$$

which show the relations among the reflection coefficients. The jump matrix $G_{0}$ carries all important scattering data generated from the associated scattering matrix $S(\lambda)$.

\subsection{Time Evolution of the Scattering Data}

In order to present the inverse scattering transforms for the nonlocal reverse-spacetime $\mathrm{mKdV}$ hierarchies, we take the $t$-derivative of the scattering relation (53) to obtain

$$
\psi_{t}^{-} E=\psi_{t}^{+} E S(\lambda)+\psi^{+} E S_{t}(\lambda),
$$

and apply the temporal spectral problems for $\psi^{ \pm}$:

$$
\psi_{t}^{ \pm}=i \lambda^{2 m+1}\left[\Omega, \psi^{ \pm}\right]+\check{Q} \psi^{ \pm} .
$$

In this way, ones can easily see that the scattering matrix $S(\lambda)$ satisfies an evolution rule:

$$
S_{t}(\lambda)=i \lambda^{2 m+1}[\Omega, S(\lambda)],
$$

where $\Omega=\operatorname{diag}\left(\beta_{1}, \beta_{2} I_{n}\right)$ as defined earlier. This evolution rule implies the following time evolution for the time-dependent scattering coefficients:

$$
\left\{\begin{array}{l}
s_{12}=s_{12}(0, \lambda) \mathrm{e}^{i \beta \lambda^{2 m+1} t}, s_{13}=s_{13}(0, \lambda) \mathrm{e}^{i \beta \lambda^{2 m+1} t}, \cdots, s_{1, n+1}=s_{1, n+1}(0, \lambda) \mathrm{e}^{i \beta \lambda^{2 m+1} t}, \\
s_{21}=s_{21}(0, \lambda) \mathrm{e}^{-i \beta \lambda^{2 m+1} t}, s_{31}=s_{31}(0, \lambda) \mathrm{e}^{-i \beta \lambda^{2 m+1} t}, \cdots, s_{n+1,1}=s_{n+1,1}(0, \lambda) \mathrm{e}^{-i \beta \lambda^{2 m+1} t},
\end{array}\right.
$$

where $\beta=\beta_{1}-\beta_{2}$ as defined before, and exhibits that all other scattering coefficients do not depend on the time variable $t$.

\subsection{Gelfand-Levitan-Marchenko Type Integral Equations}

In order to get Gelfand-Levitan-Marchenko type integral equations for the unimodular generalized matrix Jost functions, as usual let us turn the Riemann-Hilbert problems in (64) into

$$
\left\{\begin{array}{l}
G^{+}-G^{-}=G^{-} v, v=G_{0}-I_{n+1}, \lambda \in \mathbb{R}, \\
G^{ \pm} \rightarrow I_{n+1} \text { when } \lambda \in \overline{\mathbb{C}}^{ \pm} \rightarrow \infty .
\end{array}\right.
$$


Set $G(\lambda)=G^{ \pm}(\lambda)$ when $\lambda \in \mathbb{C}^{ \pm}$. We also suppose that $G$ has $R$ simple poles off $\mathbb{R}$ : $\left\{\mu_{j} \in \mathbb{C} \backslash \mathbb{R}\right\}_{j=1}^{R}$, where $R \geq 1$ is an arbitrary integer. We define

$$
\tilde{G}^{ \pm}(\lambda)=G^{ \pm}(\lambda)-\sum_{j=1}^{R} \frac{G_{j}}{\lambda-\mu_{j}}, \lambda \in \overline{\mathbb{C}}^{ \pm}, \tilde{G}(\lambda)=\tilde{G}^{ \pm}(\lambda), \lambda \in \mathbb{C}^{ \pm},
$$

in which $G_{j}$ denotes the residue of the matrix $G$ at $\lambda=\mu_{j}$, namely, $G_{j}=\operatorname{res}\left(G(\lambda), \mu_{j}\right)=$ $\lim _{\lambda \rightarrow \mu_{j}}\left(\lambda-\mu_{j}\right) G(\lambda), 1 \leq j \leq R$. Obviously, we have

$$
\left\{\begin{array}{l}
\tilde{G}^{+}-\tilde{G}^{-}=G^{+}-G^{-}=G^{-} v, \lambda \in \mathbb{R}, \\
\tilde{G}^{ \pm} \rightarrow I_{n+1} \text { when } \lambda \in \overline{\mathbb{C}}^{ \pm} \rightarrow \infty .
\end{array}\right.
$$

Utilizing the Sokhotski-Plemelj formula [25], we get the following solutions

$$
\tilde{G}(\lambda)=I_{n+1}+\frac{1}{2 \pi i} \int_{-\infty}^{\infty} \frac{\left(G^{-} v\right)(\xi)}{\xi-\lambda} d \xi
$$

for the above transformed Riemann-Hilbert problems.

Further, evaluating the limit when $\lambda \rightarrow \mu_{l}$ engenders

$$
\text { lhs }=\lim _{\lambda \rightarrow \mu_{l}} \tilde{G}=F_{l}-\sum_{j \neq l}^{R} \frac{G_{j}}{\mu_{l}-\mu_{j}}, \text { rhs }=I_{n+1}+\frac{1}{2 \pi i} \int_{-\infty}^{\infty} \frac{\left(G^{-} v\right)(\xi)}{\xi-\mu_{l}} d \xi,
$$

where $F_{l}=\lim _{\lambda \rightarrow \mu_{l}}\left[\left(\lambda-\mu_{l}\right) G(\lambda)-G_{l}\right] /\left(\lambda-\mu_{l}\right)$, and as a consequence, one obtains the required Gelfand-Levitan-Marchenko type integral equations:

$$
\begin{aligned}
I_{n+1}-F_{l}+ & \sum_{j \neq l}^{R} \frac{G_{j}}{\mu_{l}-\mu_{j}}+\frac{1}{2 \pi i} \int_{-\infty}^{\infty} \frac{\left(G^{-} G_{0}\right)(\xi)}{\xi-\mu_{l}} d \xi \\
& -\frac{1}{2 \pi i} \int_{-\infty}^{\infty} \frac{G^{-}(\xi)}{\xi-\mu_{l}} d \xi=0,1 \leq l \leq R .
\end{aligned}
$$

All these integral equations form a system to determine solutions for the resulting Riemann-Hilbert problems, and thus, the required generalized matrix Jost functions.

\subsection{Recovery of the Potential}

We finally determine how to retrieve the potential matrix, $P$, from the obtained generalized matrix Jost functions. To the end, we observe the following asymptotic behavior of $G^{+}$:

$$
G^{+}(x, t, \lambda)=I_{n+1}+\frac{1}{\lambda} G_{1}^{+}(x, t)+\mathrm{O}\left(\frac{1}{\lambda^{2}}\right), \lambda \rightarrow \infty .
$$

Inserting this asymptotic expansion into the spatial matrix spectral problem (43) and comparing constant $\left(\lambda^{0}\right)$ terms generates

$$
P=\lim _{\lambda \rightarrow \infty} \lambda\left[G^{+}(\lambda), \Lambda\right]=-\left[\Lambda, G_{1}^{+}\right] .
$$

Precisely, it yields the potential matrix:

$$
P=\left[\begin{array}{ccccc}
0 & -\alpha\left(G_{1}^{+}\right)_{12} & -\alpha\left(G_{1}^{+}\right)_{13} & \cdots & -\alpha\left(G_{1}^{+}\right)_{1, n+1} \\
\alpha\left(G_{1}^{+}\right)_{21} & 0 & 0 & \cdots & 0 \\
\alpha\left(G_{1}^{+}\right)_{31} & 0 & 0 & \cdots & 0 \\
\vdots & \vdots & \vdots & \ddots & \vdots \\
\alpha\left(G_{1}^{+}\right)_{n+1,1} & 0 & 0 & \cdots & 0
\end{array}\right]
$$


in which $G_{1}^{+}=\left(\left(G_{1}^{+}\right)_{j l}\right)_{(n+1) \times(n+1)}$. That is, the $2 n$ potential functions, $p_{j}$ and $q_{j}, 1 \leq j \leq n$, defined by

$$
p_{j}=-\alpha\left(G_{1}^{+}\right)_{1, j+1}, q_{j}=\alpha\left(G_{1}^{+}\right)_{j+1,1}, 1 \leq j \leq n,
$$

present solutions to the AKNS equations with $r=2 m+1$ in (23). When the nonlocal symmetric reduction requirement (30), which is equivalent to

$$
\left(G_{1}^{+}(-x,-t)\right)^{\dagger}=C G_{1}^{+}(x, t) C^{-1},
$$

is achieved, the reduced potential functions, $p_{j}, 1 \leq j \leq n$, present solutions to the nonlocal complex reverse-spacetime PT-symmetric mKdV hierarchies in (36).

To conclude, all this creates an algorithm for computing the required inverse scattering transforms, going from the scattering matrix $S(\lambda)$, through the solution $\left\{G^{+}(\lambda), G^{-}(\lambda)\right\}$ of the associated Riemann-Hilbert problems with the jump matrix $G_{0}(\lambda)$, to the potential matrix $P$, which present solutions to the nonlocal complex reverse-spacetime PT-symmetric $\mathrm{mKdV}$ hierarchies in (36).

\section{Soliton Solutions}

\subsection{Basic Formulation of Solutions}

Recall that the determinants of the two generalized matrix Jost functions are given in (61). Owing to $\operatorname{det} S=1$, we have

$$
\operatorname{det} T^{-}=\hat{s}_{11}=\left(S^{-1}\right)_{11}=\left|\begin{array}{cccc}
s_{22} & s_{23} & \cdots & s_{2, n+1} \\
s_{32} & s_{33} & \cdots & s_{3, n+1} \\
\vdots & \vdots & \ddots & \vdots \\
s_{n+1,2} & s_{n+1,3} & \cdots & s_{n+1, n+1}
\end{array}\right| .
$$

Let $N$ be another arbitrary natural number. Suppose that $s_{11}$ has zeros $\left\{\lambda_{k} \in \mathbb{C}\right.$, $1 \leq k \leq N\}$ and $\hat{s}_{11}$ has zeros $\left\{\hat{\lambda}_{k} \in \mathbb{C}, 1 \leq k \leq N\right\}$. In order to compute soliton solutions, we also suppose that all those zeros are geometrically simple. Thus, each of $\operatorname{ker} T^{+}\left(\lambda_{k}\right)$, $1 \leq k \leq N$, has just one single basis column vector, denoted by $v_{k}, 1 \leq k \leq N$; and each of $\operatorname{ker} T^{-}\left(\hat{\lambda}_{k}\right), 1 \leq k \leq N$, one single basis row vector, denoted by $\hat{v}_{k}, 1 \leq k \leq N$, which are determined by

$$
T^{+}\left(\lambda_{k}\right) v_{k}=0, \hat{v}_{k} T^{-}\left(\hat{\lambda}_{k}\right)=0, \text { where } 1 \leq k \leq N .
$$

It is known that one can explicitly solve the Riemann-Hilbert problems defined by (64), whose jump matrix is the identity and which possess the canonical normalization conditions in (68) and the zero structures indicated in (82) (see, e.g., [19,36]), if

$$
\lambda_{k} \neq \lambda_{l}, 1 \leq k \neq l \leq N ; \hat{\lambda}_{k} \neq \hat{\lambda}_{l}, 1 \leq k \neq l \leq N ; \text { and } \lambda_{l} \neq \hat{\lambda}_{k}, 1 \leq k, l \leq N
$$

The first two conditions above simply mean that the algebraic multiplicities of zeros of $\operatorname{det} T^{+}$and $\operatorname{det} T^{-}$are all equal to one. Based on solutions to the Riemann-Hilbert problems, it is then straightforward to determine the potential matrix $P$.

Soliton solutions are associated with the specific Riemann-Hilbert problems with $G_{0}=I_{n+1}$, the identity jump matrix. The condition of $G_{0}=I_{n+1}$ can be achieved if we assume that $s_{j 1}=\hat{s}_{1 j}=0,2 \leq j \leq n+1$. This says that only zero reflection coefficients are taken in the corresponding scattering problem. It can be proved that solutions to this kind of special Riemann-Hilbert problems can be presented as follows:

$$
G^{+}(\lambda)=I_{n+1}-\sum_{k, l=1}^{N} \frac{v_{k}\left(M^{-1}\right)_{k l} \hat{v}_{l}}{\lambda-\hat{\lambda}_{l}},\left(G^{-}\right)^{-1}(\lambda)=I_{n+1}+\sum_{k, l=1}^{N} \frac{v_{k}\left(M^{-1}\right)_{k l} \hat{v}_{l}}{\lambda-\lambda_{k}},
$$


in which $M=\left(m_{k l}\right)_{N \times N}$ denotes a square matrix, whose entries are defined by

$$
m_{k l}=\left\{\begin{array}{cl}
\frac{\hat{v}_{k} v_{l}}{\lambda_{l}-\hat{\lambda}_{k}}, & \text { if } \lambda_{l} \neq \hat{\lambda}_{k \prime} \\
0, & \text { if } \lambda_{l}=\hat{\lambda}_{k},
\end{array} \quad 1 \leq k, l \leq N,\right.
$$

for which an additional orthogonal condition

$$
\hat{v}_{k} v_{l}=0, \text { when } \lambda_{l}=\hat{\lambda}_{k}, 1 \leq k, l \leq N,
$$

is required. This condition guarantees that $\left(G^{-}\right)^{-1}(\lambda) G^{+}(\lambda)=I_{n+1}$. We remark that within this formulation of solutions, we do not need any of the three conditions in (83). If we require all those three conditions in (83), the formulation of solutions in (84) and (85) reduce to the known one in the literature (see, e.g., $[19,36]$ ).

\subsection{Nonreduced Case}

Observe that all zeros, $\lambda_{k}$ and $\hat{\lambda}_{k}, 1 \leq k \leq N$, are space and time independent and, thus, one can work out the spatial and temporal evolution laws for the eigenvectors, $v_{k}(x, t)$ and $\hat{v}_{k}(x, t), 1 \leq k \leq N$. For example, let us compute the $x$-derivative of both sides of the first set of equations in (82) as follows.

Applying (43) first and then the first set of equations in (82) again, one can arrive at

$$
T^{+}\left(x, \lambda_{k}\right)\left(\frac{d v_{k}}{d x}-i \lambda_{k} \Lambda v_{k}\right)=0,1 \leq k \leq N
$$

This property induces that for each $1 \leq k \leq N$, the vector $\frac{d v_{k}}{d x}-i \lambda_{k} \Lambda v_{k}$ must belong to the kernel of $T^{+}\left(x, \lambda_{k}\right)$, and so, a constant multiple of $v_{k}$. One can, without loss of generality, set

$$
\frac{d v_{k}}{d x}=i \lambda_{k} \Lambda v_{k}, 1 \leq k \leq N .
$$

Obviously, the time dependence for $v_{k}$ can be similarly determined by

$$
\frac{d v_{k}}{d t}=i \lambda_{k}^{2 m+1} \Omega v_{k}, 1 \leq k \leq N,
$$

by using the temporal matrix spectral problem (44). In the same way, based on the second set of equations in (82), one can determine the space and time dependence of $\hat{v}_{k}, 1 \leq k \leq N$. Therefore, we can get

$$
\begin{aligned}
& v_{k}(x, t)=\mathrm{e}^{i \lambda_{k} \Lambda x+i \lambda_{k}^{2 m+1} \Omega t} w_{k}, 1 \leq k \leq N, \\
& \hat{v}_{k}(x, t)=\hat{w}_{k} \mathrm{e}^{-i \hat{\lambda}_{k} \Lambda x-i \hat{\lambda}_{k}^{2 m+1} \Omega t}, 1 \leq k \leq N,
\end{aligned}
$$

in which $w_{k}$ and $\hat{w}_{k}, 1 \leq k \leq N$, are arbitrary constant column vectors and constant row vectors, respectively, but they need to satisfy

$$
\hat{w}_{k} w_{l}=0, \text { when } \lambda_{l}=\hat{\lambda}_{k}, 1 \leq k, l \leq N,
$$

which is just a result of (86).

Now, using the solutions in (84), one can have

$$
G_{1}^{+}=-\sum_{k, l=1}^{N} v_{k}\left(M^{-1}\right)_{k l} \hat{v}_{l},
$$


and, thus, the presentations in (80) present the $N$-soliton solution for the multicomponent AKNS equations with $r=2 m+1$ in (23):

$$
p_{j}=\alpha \sum_{k, l=1}^{N} v_{k, 1}\left(M^{-1}\right)_{k l} \hat{v}_{l, j+1}, q_{j}=-\alpha \sum_{k, l=1}^{N} v_{k, j+1}\left(M^{-1}\right)_{k l} \hat{v}_{l, 1}, 1 \leq j \leq n .
$$

\subsection{Nonlocal Case}

In order to compute soliton solutions for the nonlocal complex reverse-spacetime $\mathrm{mKdV}$ hierarchies in (36), one needs to check the involution property (81) for $G_{1}^{+}$defined by (93), which equivalently says that the potential matrix, $P$, determined by (79) needs to satisfy the reduction requirement in (30). In this way, the soliton solutions to the standard AKNS equations in (23) are reduced to the soliton solutions:

$$
p_{j}=\alpha \sum_{k, l=1}^{N} v_{k, 1}\left(M^{-1}\right)_{k l} \hat{v}_{l, j+1}, 1 \leq j \leq n,
$$

to the nonlocal complex reverse-spacetime mKdV hierarchies in (36).

In what follows, let us determine how to satisfy the involution property (81). As in the local theory (see, e.g., [31]), we first take zeros of $\operatorname{det} T^{+}(\lambda)$ (i.e., eigenvalues of the matrix spectral problems with the zero potential): $\lambda_{k} \in \mathbb{C}, 1 \leq k \leq N$, and then take $\hat{\lambda}_{k}=-\lambda_{k^{\prime}}^{*} 1 \leq k \leq N$, which define zeros of $\operatorname{det} T^{-}(\lambda)$ (i.e., eigenvalues of the adjoint matrix spectral problems with the zero potential). One one hand, we see that $\operatorname{ker} T^{+}\left(\lambda_{k}\right)$, $1 \leq k \leq N$, are spanned by

$$
v_{k}(x, t)=v_{k}\left(x, t, \lambda_{k}\right)=\mathrm{e}^{i \lambda_{k} \Lambda x+i \lambda_{k}^{2 m+1} \Omega t} w_{k}, 1 \leq k \leq N,
$$

respectively, in which $w_{k}, 1 \leq k \leq N$, are arbitrary constant column vectors. Obviously, all these column vectors $v_{k}, 1 \leq k \leq N$, defined in (96), are eigenfunctions of the matrix spectral problems with the zero potential (i.e., $u=0$ ) associated with $\lambda_{k}, 1 \leq k \leq N$. On the other hand, following the previous analysis in Section 3.1, we see that $\operatorname{ker} T^{-}\left(\lambda_{k}\right)$, $1 \leq k \leq N$, can be determined by

$$
\hat{v}_{k}(x, t)=\hat{v}_{k}\left(x, t, \hat{\lambda}_{k}\right)=\hat{w}_{k} \mathrm{e}^{-i \hat{\lambda}_{k} \Lambda x-i \hat{\lambda}_{k}^{2 m+1} \Omega t} C, 1 \leq k \leq N,
$$

respectively, in which $\hat{w}_{k}, 1 \leq k \leq N$, are arbitrary constant row vectors. It is also easy to see that all these row vectors are eigenfunctions of the adjoint matrix spectral problems with the zero potential (i.e., $u=0$ ) associated with $\hat{\lambda}_{k}, 1 \leq k \leq N$.

In order to satisfy the particular orthogonal condition (92), let us impose the conditions on $\hat{w}_{k}$ and $w_{l}, 1 \leq k, l \leq N$ :

$$
\hat{w}_{k} C w_{l}=0 \text {, when } \lambda_{l}=\hat{\lambda}_{k}, 1 \leq k \leq N .
$$

We point out that, for each $1 \leq k \leq N$, the situation that $\lambda_{k}=\hat{\lambda}_{k}$ happens, only while $\lambda_{k} \in i \mathbb{R}$ and $\hat{\lambda}_{k}=-\lambda_{k}^{*}$.

Finally, as soon as the orthogonal condition (98) is satisfied, the resulting solutions of the special Riemann-Hilbert problems, defined by (84), (85), (96) and (97), satisfy the involution property:

$$
G^{+}\left(-x,-t,-\lambda^{*}\right)=C\left(G^{-}\right)^{-1}(x, t, \lambda) C^{-1}
$$

This tells that $G_{1}^{+}$satisfies the condition (81) for the nonlocal reverse-spacetime reductions in (29). Thus the Formula (95), along with (84), (85), (96) and (97), gives rise to soliton solutions for the nonlocal complex reverse-spacetime $\mathrm{mKdV}$ hierarchies of equations in (36). 
Consider the first equation $(m=1)$ in the hierarchy of scalar nonlocal equations $(n=1)$. When $N=1$, let us take $\hat{\lambda}_{1}=-\lambda_{1}^{*}$, and denote $w_{1}=\left(w_{1,1}, w_{1,2}\right)^{T}$ and $\hat{w}_{1}=\left(\hat{w}_{1,1,}, \hat{w}_{1,2}\right)$. In this way, we get the one-soliton solution to the nonlocal complex reverse-spacetime $\mathrm{mKdV}$ Equation (40) with $n=1$ :

$$
p(x, t)=\frac{2 \alpha \operatorname{Re}\left(\lambda_{1}\right) w_{1,1} \hat{w}_{1,2} \Sigma}{w_{1,1} \hat{w}_{1,1} \mathrm{e}^{i\left(\alpha \lambda_{1}^{*}+\beta \lambda_{1}^{* 3} t\right)}+w_{1,2} \hat{w}_{1,2} \Sigma \mathrm{e}^{-i\left(\alpha \lambda_{1} x+\beta \lambda_{1}^{3} t\right)}},
$$

where $\lambda_{1} \in \mathbb{C}$ is arbitrary, and $w_{1,1}^{*} \hat{w}_{1,2}+w_{1,2}^{*} \hat{w}_{1,1}=0$, which is the condition that the involution property requires.

\section{Concluding Remarks}

The aim of the paper is to propose nonlocal complex reverse-spacetime PT-symmetric modified Korteweg-de Vries (mKdV) hierarchies, and formulate the corresponding inverse scattering transforms and $\mathrm{N}$-soliton solutions from a perspective of Riemann-Hilbert problems. A crucial step is to build Riemann-Hilbert problems, beginning with matrix spectral problems. In our formulation, solutions to the Riemann-Hilbert problems were presented by using the the Sokhotski-Plemelj formula, and the corresponding inverse scattering transforms were established through the associated Riemann-Hilbert problems. Moreover, a formulation of solutions was proposed for specific Riemann-Hilbert problems, whose jump is the identity matrix (or equivalently, the reflectionless inverse scattering transforms) and, thus, $\mathrm{N}$-soliton solutions to the nonlocal complex reverse-spacetime PTsymmetric $\mathrm{mKdV}$ hierarchies were generated by applying such a formulation of solutions for the special Riemann-Hilbert problems.

It has been gradually recognized that the Riemann-Hilbert problems are good analytic objects to build the inverse scattering transforms and construct soliton solutions to nonlinear integrable equations (see also, e.g., [20-22,37]). The corresponding technique has been already extended to treat initial-boundary value problems of nonlinear integrable equations on either the half-line or the finite interval [38,39]. Formulations of solutions, however, can differ from case to case for nonlocal reductions and correspondingly nonlocal integrable equations [8]. It would be intriguing to generate more nonlocal integrable hierarchies of either reverse-time or reverse-space or reverse-spacetime type. There also exist other efficient methods to construct soliton solutions in soliton theory, including the Wronskian technique [40,41], the Hirota direct method [42], the generalized bilinear technique [43], the Darboux transformation $[44,45]$ and the wavefunction ansatz method [46]. It will definitely be of significant importance to explore relationships among those different approaches. Particularly, it is very interesting to see how the Hirota method can be applied to a nonlocal soliton hierarchy.

We also remark that it will be very interesting to construct different classes of explicit exact solutions to both local and nonlocal nonlinear integrable equations, for instance, positon and complexiton solutions [47,48], lump solutions [49,50], algebro-geometric solutions [51,52], solitonless solutions [53,54] and dromions [55,56], through the RiemannHilbert technique. It is another interesting topic for future research to build Riemann-Hilbert problems for solving various integrable counterparts such as integrable couplings, fractional analogous soliton equations and supersymmetric soliton hierarchies. It is expected that a clear understanding could be achieved about soliton solutions to those integrable counterparts, both local and nonlocal, from a Riemann-Hilbert problem perspective.

Author Contributions: Formal analysis, W.-X.M.; Funding acquisition, L.L.; Methodology, W.-X.M.; Resources, L.L.; Validation, L.L.; Writing-original draft, W.-X.M. All authors have read and agreed to the published version of the manuscript.

Funding: The research received no external funding.

Institutional Review Board Statement: Not applicable.

Informed Consent Statement: Not applicable. 
Data Availability Statement: All data generated or analyzed during this study are included in this published article.

Acknowledgments: The work was supported in part by the National Natural Science Foundation of China under the grants 11975145, 11972291 and 11771151, the Natural Science Foundation for Colleges and Universities in Jiangsu Province (17 KJB 110020), Guagdong Natural Science Foundation (2017A030313008), Guangzhou Science and Technology Program (No. 201904010362) and the Fundamental Research Funds for the Central Universities (No. D2192580).

Conflicts of Interest: The authors declare that there is no conflict of interest.

\section{References}

1. Ablowitz, M.J.; Musslimani, Z.H. Integrable Nonlocal Nonlinear Equations. Stud. Appl. Math. 2017, 39, 7-59. [CrossRef]

2. Ablowitz, M.J.; Musslimani, Z.H. Integrable Nonlocal Nonlinear Schrödinger Equation. Phys. Rev. Lett. 2013, 110, 064105. [CrossRef]

3. Ablowitz, M.J.; Musslimani, Z.H. Inverse Scattering Transform for the Integrable Nonlocal Nonlinear Schrödinger Equation. Nonlinearity 2016, 29, 915-946. [CrossRef]

4. Gerdjikov, V.S.; Saxena, A. Complete Integrability of Nonlocal Nonlinear Schrödinger Equation. J. Math. Phys. 2017, 58, 013502. [CrossRef]

5. Ji, J.L.; Zhu, Z.N. Soliton Solutions of an Integrable Nonlocal Modified Korteweg-de Vries Equation through Inverse Scattering Transform. J. Math. Anal. Appl. 2017, 453, 973-984. [CrossRef]

6. Ablowitz, M.J.; Luo, X.D.; Musslimani, Z.H. Inverse Scattering Transform for the Nonlocal Nonlinear Schrödinger Equation with Nonzero Boundary Conditions. J. Math. Phys. 2018, 59, 011501. [CrossRef]

7. Ablowitz, M.J.; Feng, B.F.; Luo, X.D.; Musslimani, Z.H. Inverse Scattering Transform for the Nonlocal Reverse Space-time Nonlinear Schrödinger Equation. Theoret. Math. Phys. 2018, 196, 1241-1267. [CrossRef]

8. Ma, W.X. Inverse Scattering for Nonlocal Reverse-time Nonlinear Schrödinger Equations. Appl. Math. Lett. 2020, $102,106161$. [CrossRef]

9. Yang, J. General N-solitons and Their Dynamics in Several Nonlocal Nonlinear Schrödinger Equations. Phys. Lett. A 2019, 383, 328-337. [CrossRef]

10. Ma, L.Y.; Zhu, Z.N. Nonlocal Nonlinear Schrödinger Equation and Its Discrete Version: Soliton Solutions and Gauge Equivalence. J. Math. Phys. 2016, 57, 083507. [CrossRef]

11. Huang, X.; Ling, L. Soliton Solutions for the Nonlocal Nonlinear Schrödinger Equation. Eur. Phys. J. Plus 2016, 131, 148. [CrossRef]

12. Vinayagam, P.S.; Radha, R.; Al-Khawaja, U.; Ling, L. New Classes of Solutions in the Coupled PT Symmetric Nonlocal Nonlinear Schrödinger Equations with Four Wave Mixing. Commun. Nonlinear Sci. Numer. Simul. 2018, 59, 387-395. [CrossRef]

13. Liu, D.Y.; Sun, W.R. Rational Solutions for the Nonlocal Sixth-order Nonlinear Schrödinger Equation. Appl. Math. Lett. 2018, 84, 63-69. [CrossRef]

14. Yang, Y.Q.; Suzuki, T.; Cheng, X.P. Darbourx Transformations and Exact Solutions for the Integrable Nonlocal LakshmananPorsezian-Daniel Equation. Appl. Math. Lett. 2020, 99, 105998. [CrossRef]

15. Gürses, M.; Pekcan, A. Nonlocal Nonlinear Schrödinger Equations and Their Soliton Solutions. J. Math. Phys. 2018, 59, 051501. [CrossRef]

16. Gürses, M.; Pekcan, A. Nonlocal Modified KdV Equations and Their Soliton Solutions by Hirota Method. Commun. Nonlinear Sci. Numer. Simul. 2019, 67, 427-448. [CrossRef]

17. Fokas, A.S. Integrable Multidimensional Versions of the Nonlocal Nonlinear Schrödinger Equation. Nonlinearity 2016, 29, 319-324. [CrossRef]

18. Song, C.Q.; Xiao, D.M.; Zhu, Z.N. Solitons and Dynamics for a General Integrable Nonlocal Coupled Nonlinear Schrödinger Equation. Commun. Nonlinear Sci. Numer. Simul. 2017, 45, 13-28. [CrossRef]

19. Novikov, S.P.; Manakov, S.V.; Pitaevskii, L.P.; Zakharov, V.E. Theory of Solitons: The Inverse Scattering Method; Consultants Bureau: New York, NY, USA, 1984.

20. Wang, D.S.; Zhang, D.J.; Yang, J. Integrable Properties of the General Coupled Nonlinear Schrödinger Equations. J. Math. Phys. 2010, 51, 023510. [CrossRef]

21. Xiao, Y.; Fan, E.G. A Riemann-Hilbert Approach to the Harry-Dym Equation on the Line. Chin. Ann. Math. Ser. B 2016, 37, 373-384. [CrossRef]

22. Geng, X.G.; Wu, J.P. Riemann-Hilbert Approach and N-soliton Solutions for a Generalized Sasa-Satsuma Equation. Wave Motion 2016, 60, 62-72. [CrossRef]

23. Ma, W.X. Riemann-Hilbert Problems and N-soliton Solutions for a Coupled mKdV System. J. Geom. Phys. 2018, 132, 45-54. [CrossRef]

24. Ma, W.X. Application of the Riemann-Hilbert Approach to the Multicomponent AKNS Integrable Hierarchies. Nonlinear Anal. Real World Appl. 2019, 47, 1-17. [CrossRef]

25. Gakhov, F.D. Boundary Value Problems; Elsevier Science: London, UK, 2014. 
26. Ma, W.X.; Zhou, R.G. Adjoint Symmetry Constraints of Multicomponent AKNS Equations. Chin. Ann. Math. Ser. B 2002, 23, 373-384. [CrossRef]

27. Ablowitz, M.J.; Kaup, D.J.; Newell, A.C.; Segur, H. The Inverse Scattering Transform-Fourier Analysis for Nonlinear Problems. Stud. Appl. Math. 1974, 53, 249-315. [CrossRef]

28. Tu, G.Z. On Liouville Integrability of Zero-curvature Equations and the Yang Hierarchy. J. Phys. A Math. Gen. 1989, $22,2375-2392$.

29. Ma, W.X.; Chen, M. Hamiltonian and Quasi-Hamiltonian Structures Associated with Semi-direct Sums of Lie Algebras. J. Phys. A Math. Gen. 2006, 39, 10787-10801. [CrossRef]

30. Ma, W.X.; Zhou, R.G. Adjoint Symmetry Constraints Leading to Binary Nonlinearization. J. Nonlinear Math. Phys. 2002, 9 (Suppl. 1), 106-126. [CrossRef]

31. Ma, W.X. Riemann-Hilbert Problems and Soliton Solutions of a Multicomponent mKdV System and Its Reduction. Math. Meth. Appl. Sci. 2019, 42, 1099-1113. [CrossRef]

32. Gerdjikov, V.S. Geometry, Integrability and Quantization. In Proceedings of the 6th International Conference, Varna, Bulgaria, 3-10 June 2004; Softex: Sofia, Bulgaria, 2005; pp. 78-125.

33. Doktorov, E.V.; Leble, S.B. A Dressing Method in Mathematical Physics; Springer: Dordrecht, The Netherlands, 2007.

34. Ma, W.X.; Yong, X.L.; Qin, Z.Y.; Gu, X.; Zhou, Y. A Generalized Liouville's Formula. Appl. Math. Ser. B 2017, preprint.

35. Beals, R.; Coifman, R.R. Scattering and Inverse Scattering for First Order Systems. Comm. Pure Appl. Math. 1984, 37, 39-90. [CrossRef]

36. Kawata, T. Riemann Spectral Method for the Nonlinear Evolution Equation. In Advances in Nonlinear Waves; Pitman: Boston, MA, USA, 1984; Volume I, pp. 210-225.

37. Yang, J. Nonlinear Waves in Integrable and Nonintegrable Systems; SIAM: Philadelphia, PA, USA, 2010.

38. Fokas, A.S.; Lenells, J. The Unified Method: I. Nonlinearizable Problems on the Half-line. J. Phys. A Math. Theor. 2012, 45, 195201. [CrossRef]

39. Fokas, A.S.; Lenells, J. The Unified Method: III. Nonlinearizable Problems on the Interval. J. Phys. A Math. Theor. 2012, 45, 195203. [CrossRef]

40. Freeman, N.C.; Nimmo, J.J.C. Soliton Solutions of the Korteweg-de Vries and Kadomtsev-Petviashvili Equations: The Wronskian Technique. Phys. Lett. A 1983, 95, 1-3. [CrossRef]

41. Ma, W.X.; You, Y. Solving the Korteweg-de Vries Equation by Its Bilinear Form: Wronskian Solutions. Trans. Am. Math. Soc. 2005, 357, 1753-1778. [CrossRef]

42. Hirota, R. The Direct Method in Soliton Theory; Cambridge University Press: New York, NY, USA, 2004.

43. Ma, W.X. Generalized Bilinear Differential Equations. Stud. Nonlinear Sci. 2011, 2, 140-144.

44. Matveev, V.B.; Salle, M.A. Darboux Transformations and Solitons; Springer: Berlin, Germany, 1991.

45. Ma, W.X.; Zhang, Y.J. Darboux Transformations of Integrable Couplings and Applications. Rev. Math. Phys. 2018, $30,1850003$. [CrossRef]

46. Zafar, A.; Seadawy, A.R. The Conformable Space-time Fractional mKdV Equations and Their Exact Solutions. J. King Saud Univ. Sci. 2019, 31, 1478-1484. [CrossRef]

47. Matveev, V.B. Generalized Wronskian Formula for Solutions of the KdV Equations: First Applications. Phys. Lett. A 1992, 166, 205-208. [CrossRef]

48. Ma, W.X. Complexiton Solutions to the Korteweg-de Vries Equation. Phys. Lett. A 2002, 301, 35-44. [CrossRef]

49. Ma, W.X.; Zhou, Y. Lump Solutions to Nonlinear Partial Differential Equations via Hirota Bilinear Forms. J. Differ. Equ. 2018, 264, 2633-2659. [CrossRef]

50. Ma, W.X.; Zhang, L.Q. Lump Solutions with Higher-order Rational Dispersion Relations. Pramana J. Phys. 2020, 94, 43. [CrossRef]

51. Belokolos, E.D.; Bobenko, A.I.; Enol'skii, V.Z.; Its, A.R.; Matveev, V.B. Algebro-Geometric Approach to Nonlinear Integrable Equations; Springer: Berlin, Germany, 1994.

52. Ma, W.X. Trigonal Curves and Algebro-geometric Solutions to Soliton Hierarchies I, II. Proc. R. Soc. A 2017, 473, 20170232-20170233. [CrossRef] [PubMed]

53. Ma, W.X. Long-time Asymptotics of a Three-component Coupled mKdV System. Mathematics 2019, 7, 573. [CrossRef]

54. Ma, W.X. Long-time Asymptotics of a Three-component Coupled Nonlinear Schrödinger System. J. Geom. Phys. 2020, 153, 103669. [CrossRef]

55. Boiti, M.; Leon, J.J.P.; Martina, L.; Pempinelli, F. Scattering of Localized Solitons in the Plane. Phys. Lett. A 1988, 132, 432-439. [CrossRef]

56. Hietarinta, J. One-dromion Solutions for Generic Classes of Equations. Phys. Lett. A 1990, 149, 113-118. [CrossRef] 